\title{
Stationäre Therapie der komplexen PTBS in Folge körperlicher oder sexualisierter Gewalt in der Kindheit: Wirksamkeit und Prädiktoren des Behandlungsverlaufs
}

\section{Inpatient Treatment of Complex PTSD Following Childhood Abuse: Effectiveness and Predictors of Treatment Outcome}

Autoren

Leonhard Kratzer', Peter Heinz¹, Rebecca Schennach'2,3, Günter Karl Schiepek ${ }^{4,5,6}$, Frank Padberg ${ }^{3}$, Andrea Jobst ${ }^{3}$

Institute

1 Abteilung für Psychotraumatologie, Klinik St. Irmingard, Prien am Chiemsee

2 Schön Klinik Roseneck, Psychosomatik, Prien am Chiemsee

3 Klinik und Poliklink für Psychiatrie und Psychotherapie, Ludwig-Maximilians-Universität, München

4 Institut für Synergetik und Psychotherapieforschung, Paracelsus Medizinische Privatuniversität, Salzburg/ Österreich

5 Universitätsklinik für Psychiatrie und Psychotherapie, Paracelsus Medizinische Privatuniversität, Salzburg/ Österreich

6 Institut für Psychologie, Ludwig-Maximilians-Universität, München

Schlüsselwörter

Posttraumatische Belastungsstörung, Eye Movement Desensitization and Reprocessing (EMDR), Sexueller Missbrauch, Körperliche Misshandlung, klinische Signifikanz

Key words

Post-traumatic Stress Disorder, Eye Movement Desensitization and Reprocessing, childhood sexual abuse, childhood physical abuse, clinical significance

eingereicht $\quad 04.12 .2017$

akzeptiert $\quad 19.02 .2018$

Bibliografie

DOI https://doi.org/10.1055/a-0591-3962

Online-Publikation: 30.5.2018

Psychother Psych Med 2019; 69: 114-122

(c) Georg Thieme Verlag KG Stuttgart · New York

ISSN 0937-2032

Korrespondenzadresse

Leonhard Kratzer

Klinik St. Irmingard

Abteilung für Psychotraumatologie

Osternacher Straße 103

83209 Prien am Chiemsee

I.kratzer@st-irmingard.de

\section{ZUSAMMENFASSUNG}

Hintergrund Die Wirksamkeit stationärer traumafokussierter Psychotherapie ist bei PatientInnen mit komplexer posttraumatischer Belastungsstörung (PTBS) insbesondere unter klinischen Routinebedingungen unzureichend belegt. Ziel dieser Untersuchung war neben der Verlaufsanalyse die Identifikation von Prädiktoren des Behandlungserfolges.

Methode 150 PatientInnen der Abteilung für Psychotraumatologie der Klinik St. Irmingard mit komplexer PTBS in Folge körperlicher oder sexualisierter Gewalt in der Kindheit beantworteten Fragebögen zu PTBS, Kindheitstraumata, Achtsamkeit, Dissoziation und allgemeiner Psychopathologie. Die Unterschiede zwischen Prä- und Postmessung wurden regressionsanalytisch untersucht. Mittels konditionalem Klassifikationsbaum wurde untersucht, welche Parameter eine Response prädizieren. Ergebnisse Die signifikante Verbesserung der PTBS-Symptomatik entsprach einem großen Effekt $(d=1,8)$ und einer Responserate von $52 \%$ gemäß Reliable Change Index $(p<0,05)$. Hinsichtlich anderer Symptombereiche waren Verbesserungen in der Größenordnung mittlerer bis großer Effekte $(0,5<\mathrm{d}<1,1)$ zu beobachten. Sowohl Abbrüche (7\%) als auch Verschlechterungen (4\%) waren selten. Somatoforme Beschwerden, das Vorliegen einer komplexen dissoziativen Störung sowie Achtsamkeitsdefizite erwiesen sich als negative Prädiktoren einer reliablen Besserung der PTBS-Belastung.

Diskussion und Schlussfolgerung Traumafokussierte Psychotherapie ist unter naturalistischen Bedingungen bei PatientInnen mit komplexer PTBS sicher und wirksam durchführbar. Zugleich zeigen sich hohe Nonresponseraten. Zukünftige Untersuchungen sollten die Bedeutung der identifizierten Prädiktoren für Nonresponse näher untersuchen. Mögliche Maßnahmen zur Reduktion von Nonresponse werden diskutiert.

\section{ABSTRACT}

Background There is a lack of studies investigating the effectiveness of inpatient trauma-focused psychotherapy of complex post-traumatic stress disorder. The first aim of this retrospective investigation was to analyze the course of PTSD. Second, possible predictors of treatment response were investigated.

Methods 150 inpatients of Clinic St. Irmingard with complex PTSD following childhood physical and childhood sexual abuse 
were assessed regarding childhood abuse, PTSD symptomatology, mindfulness, dissociation and general psychopathology. Differences in pre and post scores were analyzed using regression analyses. A classification tree was used to identify predictors of response.

Results The significant reduction of PTSD symptoms corresponded to a large effect $(d=1.8)$ and a reponse rate of $52 \%$ according to the reliable change index $(p<0.05)$. Effect sizes for other symptoms were medium to large $(0.5<\mathrm{d}<1.1)$ and both drop-outs (7\%) and worsenings (4\%) were scarce. Soma- toform symptoms, complex dissociative disorders and mindfulness deficits were identified as negative predictors of reliable change.

Conclusions Trauma-focused inpatient treatment is safe and effective for patients with complex PTSD under naturalistic conditions. Yet, despite significant improvements there is a high rate of nonresponse. Future studies should further investigate the negative predictors of treatment outcome we identified. Possible ways to reduce nonresponse are discussed.

\section{Hintergrund}

\section{Die komplexe posttraumatische Belastungsstörung}

Posttraumatische Belastungsstörungen in Folge lange andauernder oder wiederholter interpersoneller Gewalt führen oftmals zu einem komplexen Störungsbild, das über die in der ICD-10 [1] beschriebenen Kriterien der PTBS hinausgeht und durch zusätzliche somatoforme und dissoziative Symptome, Affektregulationsstörungen, interaktionelle Störungen und Störungen des Selbstkonzeptes geprägt ist. Diese „komplexe PTBS“ [2] wird heute zumeist als das parallele Vorliegen der Kriterien der Disorder of Extreme Stress Not Otherwise Specified (DESNOS) [3] und der PTBS-Kriterien operationalisiert [4]. Trotz überlappender Kriterien der komplexen PTBS und der Borderline-Persönlichkeitsstörung handelt es sich um signifikant abgegrenzte Entitäten [5]. In der ICD-11 wird die komplexe PTBS voraussichtlich als PTBS mit zusätzlichen Störungen der Emotionsregulation, des Selbstkonzeptes und der Beziehungsfähigkeit enthalten sein [6].

Durch den häufigen Ausschluss von Patientlnnen mit komplexer PTBS aus randomisiert-kontrollierten Studien [7] und die fehlende Erfassung bedeutsamer Symptombereiche der komplexen PTBS fehlten lange Zeit klare Befunde zur Wirksamkeit eines traumafokussierten Vorgehens bei komplexer PTBS [8]. Gleichzeitig wurde immer wieder auf hohe Abbruchraten und ein schlechteres Ansprechen auf Therapie der PatientInnen mit komplexer PTBS hingewiesen $[9,10]$. In der Folge entstand insbesondere im deutschsprachigen Raum eine kritische Haltung gegenüber einer konfrontativen Bearbeitung traumatischer Erinnerungen zugunsten einer „Stabilisierung“ der PatientInnen [11, 12].

\section{Zur Rolle von „Stabilisierung“}

Die Evidenz für ein stabilisierendes Vorgehen ist limitiert und weist nur geringe Effekte auf [13]. Auch kommt es infolge stabilisierender stationärer Behandlungen in den seltensten Fällen zu einer traumakonfrontativen Behandlung [14]. Damit wird PatientInnen mit komplexer PTBS oftmals ein wirksamer Behandlungsbaustein vorenthalten, ohne dass es dafür ausreichende empirische Evidenz gäbe [15]. Im Gegenteil konnten die Bedenken gegen ein traumafokussiertes Vorgehen in der Behandlung der komplexen PTBS in jüngerer Vergangenheit als Mythen entlarvt werden. So konnte gezeigt werden, dass ein traumakonfrontatives Vorgehen auch bei komplexen posttraumatischen Belastungsstörungen, komorbiden
Persönlichkeitsstörungen, selbstverletzendem Verhalten und sogar komorbider Psychose sicher und wirksam durchführbar ist [16-18]. Traumafokussierte Psychotherapie ist auch bei komplexer PTBS wirksamer als nicht traumafokussierte Psychotherapie und geht entgegen entsprechenden Befürchtungen nicht mit höheren Abbruchraten einher [19].

Gleichzeitig empfiehlt die Mehrzahl der ExpertInnen heute, das traumafokussierte Vorgehen bei PatientInnen mit komplexer PTBS, komorbider Borderline-Störung oder komplexen dissoziativen Störungen wie der Dissoziativen Identitätsstörung (DIS) oder der nicht näher bezeichneten dissoziativen Störung (DDNOS) [20] mit einem Training der Emotionsregulation zu kombinieren und in der Durchführung anzupassen [13, 16, 17, 21]. Cloitre et al. [22] konnten zeigen, dass durch ein derartig angepasstes Vorgehen signifikant bessere Behandlungsergebnisse zu erzielen sind.

\section{Fragestellungen}

Im Rahmen der vorliegenden retrospektiven Untersuchung sollten die Symptomverläufe von PatientInnen mit komplexer PTBS nach schwerer körperlicher oder sexualisierter Gewalt in der Kindheit im Rahmen einer auf Traumakonfrontation und Fertigkeitenaufbau ausgerichteten stationären Behandlung analysiert werden. Darüber hinaus sollten mögliche Prädiktoren des Behandlungsverlaufes identifiziert werden.

\section{Methode}

\section{Behandlungskonzept der Abteilung für Psychotraumatologie der Klinik St. Irmingard}

Das Behandlungskonzept der Abteilung für Psychotraumatologie der Klinik St. Irmingard [23] versucht die oben genannten Befunde durch ein auf Traumakonfrontation einerseits, und Fertigkeitenaufbau mittels spezifischer Skillsgruppen [24, 25] andererseits ausgerichtetes Vorgehen zu integrieren. Anders als in der DBT-PTSD [16] erfolgt die Traumakonfrontation nicht durch imaginatives Wiedererleben, sondern mittels EMDR [26]. In Abgrenzung von einer rein eklektischen Kombination „stabilisierender“ und konfrontativer Interventionen versteht sich das Behandlungskonzept als „Destabilisierung im Kontext von Stabilität“ [27]. „Stabilisierung“ schafft in diesem dialektischen Verständnis die Rahmenbedingungen und Voraussetzungen für destabilisierende Prozesse wie die 
konfrontative Bearbeitung traumatischer Erinnerungen. Tägliches Prozessmonitoring stellt sicher, dass allgemeine Wirkfaktoren wie eine gute therapeutische Beziehung und Veränderungsmotivation jederzeit in ausreichendem Maße realisiert sind [28].

Alle PatientInnen erhalten pro Woche 100-150 Min psychotherapeutische sowie 50 Min pflegerische Einzelgespräche. Die Traumakonfrontation mittels EMDR umfasst meist fünf bis zehn Behandlungsstunden. Darüber hinaus nehmen die PatientInnen wöchentlich an Gruppenangeboten zur Verbesserung der Stressregulation (75 Min.), der Emotionsregulation (75 Min.), der Achtsamkeit (100200 Min.) sowie sozialer Kompetenzen (100 Min.) teil. Ergänzend bestehen Angebote zur Psychoedukation (75 Min.), Kunsttherapie (120 Min.), Körpertherapie (75 Min.), Imagination (25 Min.), Ressourcenaktivierung (100 Min.), Yoga (100 Min.), Qi Gong (50 Min.), Sozialberatung sowie ein umfassendes physiotherapeutisches Angebot. Die Einzel- und Gruppentherapien werden durch Psychologische Psychotherapeutlnnen und FachärztInnen für Psychiatrie und/oder Psychosomatische Medizin durchgeführt. Alle Einzeltherapeutlnnen sind zertifizierte EMDR-TherapeutInnen oder befinden sich in fortgeschrittener, engmaschig supervidierter Fortbildung. Interne und externe Fall- und Teamsupervisionen stellen eine hohe Manualtreue sicher. Für fallbezogene Besprechungen stehen wöchentlich mindestens 200 Min zur Verfügung.

Da die Behandlung der komplexen PTBS langfristig angelegte, integrierte Behandlungspläne erfordert und primär ambulant zu leisten ist, werden nur PatientInnen mit ambulanter Psychotherapie aufgenommen. Stets erfolgt eine enge Abstimmung mit den ambulanten BehandlerInnen, die über Fragebögen in die Anmeldung, Indikationsprüfung und Behandlungsplanung involviert sind. Zudem wird die Indikation in einem Vorgespräch überprüft.

Auch die aktive Bahnung eines Transfers von Therapieerfolgen in den Alltag nimmt einen hohen Stellenwert ein und wird durch die standardisierte Erarbeitung konkreter Ziele für die Zeit nach der Entlassung sowie regelmäßige Belastungserprobungen im häuslichen Umfeld unterstützt. Bei Bedarf werden Fallkonferenzen mit dem ambulanten Psychotherapeuten durchgeführt und Kontakt zu ergänzenden Unterstützungsangeboten wie Tagesstätten, therapeutischen Wohngruppen oder dem Sozialpsychiatrischen Dienst hergestellt.

Auf der Ebene konkreter Interventionen spielen Überlegungen, wie das traumakonfrontative Vorgehen an die Bedürfnisse von PatientInnen mit komplexer PTBS angepasst werden kann, eine zentrale Rolle. So kommen sowohl Techniken der „skills-assisted exposure“ [16] als auch Modifikationen der EMDR-Methode [21] zum Einsatz. Besonderes Augenmerk gilt dabei auch Phobien und Vermeidungsstrategien, die im Vorfeld der Konfrontation des Indextraumas durch spezifische Techniken reduziert werden können.

\section{PatientInnen und Ablauf der Untersuchung}

Die vorliegende retrospektive Analyse von Therapieverläufen beruht auf den Daten von 150 PatientInnen, die im Zeitraum von Juni 2013 bis Dezember 2016 in der Abteilung für Psychotraumatologie der Klinik St. Irmingard im Mittel 74,8 Tage (SD = 15,2; $\min =49$; max $=98$ ) behandelt wurden. Die testdiagnostischen Untersuchungen mittels revidierter Impact-of-Event-Scale (IES-R), Dissociative Experiences Scale - Taxon (DES-T), Freiburger Fragebogen zur Achtsamkeit (FFA) sowie der Hamburger Module zur Erfassung allge- meiner Aspekte psychosozialer Gesundheit für die therapeutische Praxis (HEALTH-49) erfolgten jeweils innerhalb von 2 Tagen nach Aufnahme ( $\mathrm{t} 1$ ) beziehungsweise vor Entlassung ( $\mathrm{t} 2$ ). Das Interview zur komplexen posttraumatischen Belastungsstörung (I-kPTBS) sowie der strukturierte Interviewleitfaden zur Diagnose dissoziativer Störungen (SIDDS) wurden innerhalb der ersten zwei Behandlungswochen durch die zuständige Einzeltherapeutin durchgeführt. Die Diagnosen der PTBS sowie komorbider Störungen wurden anhand der ICD-10 Forschungskriterien [1] gestellt. Als Einschlusskriterien für die vorliegende Untersuchung wurden das Vorliegen einer PTBS gemäß ICD-10 [1] bezogen auf einen schweren körperlichen oder sexuellen Missbrauch in der Kindheit, das Vorliegen einer DESNOS sowie die schriftliche Zustimmung zur anonymisierten wissenschaftlichen Auswertung therapiebezogener Daten definiert. Im Sinne einer hohen externen Validität wurden keine Ausschlusskriterien festgelegt. Nicht eingeschlossen wurden die Daten von PatientInnen, die im betreffenden Zeitraum anlässlich einer vierwöchigen „Probetherapie“ aufgenommen wurden. Die Daten von elf PatientInnen (6,8\%), die im betreffenden Zeitraum behandelt wurden und die Einschlusskriterien erfüllten, aber ihren Aufenthalt abbrachen oder disziplinarisch entlassen wurden, gingen nicht in die Untersuchungsstichprobe ein. Die Untersuchung wurde im Einklang mit nationalem Recht sowie gemäß der Deklaration von Helsinki von 1975 (in der aktuellen, überarbeiteten Fassung) durchgeführt.

\section{Instrumente}

\section{Childhood Trauma Questionnaire (CTQ)}

Der CTQ [29] ist ein Selbstbeurteilungsinstrument mit 34 Items und dient der retrospektiven Erfassung traumatischer Erfahrungen in der Kindheit. Mit je 5 Items werden sexueller Missbrauch, physischer Missbrauch, physische Vernachlässigung, emotionaler Missbrauch und emotionale Vernachlässigung erfasst. Die deutsche Übersetzung zeigt gute interne Konsistenzen [30].

\section{Impact-of-Event Scale - Revised}

Die IES-R [31] wurde von Maercker und Schützwohl [32] übersetzt und validiert. Das Selbstbeurteilungsinstrument besteht aus 22 Items, mittels derer die Skalen Intrusion, Vermeidung und Hyperarousal erfasst werden. Die Items werden auf vierstufigen LikertSkalen beantwortet. Die IES-R ist ein valides, änderungssensitives Verfahren mit insgesamt zufriedenstellenden psychometrischen Eigenschaften [33]. In der vorliegenden Untersuchung wurde die IES-R von den PatientInnen in Bezug auf alle traumatischen Erinnerungen ausgefüllt.

\section{Freiburger Fragebogen zur Achtsamkeit}

Die Langform des FFA [34] erfasst mit 30 Items reliabel $(\alpha=0,92)$ einen allgemeinen Faktor der Achtsamkeit. Die Items werden auf vierstufigen Likert-Skalen beantwortet.

\section{Dissociative Experiences Scale - Taxon}

Die deutsche Fassung der DES-T [35] wurde 2015 validiert [36]. Das Selbstbeurteilungsinstrument erfasst mit 8 Items, die mittels visueller Analogskalen beantwortet werden, reliabel $(\alpha=0,92)$ pathologische Dissoziation. 
Hamburger Module zur Erfassung allgemeiner Aspekte psychosozialer Gesundheit für die therapeutische Praxis

Als allgemeines Maß psychosozialer Gesundheit kam die HEALTH-49 [37] zum Einsatz. Die HEALTH-49 erfasst mit 49 Items die zehn Skalen Somatoforme Beschwerden, Depressivität, Phobische Ängste, Psychische und somatoforme Beschwerden, Psychisches Wohlbefinden, Interaktionelle Schwierigkeiten, Selbstwirksamkeit, Aktivität und Partizipation, Soziale Unterstützung und Soziale Belastung. Die Bearbeitung der Items erfolgt über 5-stufige Likert-Skalen. Das Verfahren ist als valide und änderungssensitiv zu bewerten und weist mit Ausnahme der Subskala Soziale Belastung hohe interne Konsistenzen auf [37].

\section{Interview zur komplexen posttraumatischen Belastungsstörung}

Das I-kPTBS [38] stellt die deutsche Übersetzung des Structured Interview for Disorders of Extreme Stress (SIDES) von Pelcovitz et al. [3] dar. Das Interview umfasst 27 Unterkriterien, mittels derer das Vorliegen der 6 DESNOS-Kriterien erfasst wird. Die interne Konsistenz liegt bei $\alpha=0,88$.

Strukturierter Interviewleitfaden zur Diagnose dissoziativer Störungen

Das SIDDS von Overkamp [39] stellt eine Übersetzung der Dissociative Disorders Interview Schedule (DDIS) von Ross [40] dar. Es erlaubt neben der Diagnose einer DDNOS auch die Diagnose einer DIS mit einer Interrater-Reliabilität von 0,95 und einer Sensitivität von $94,4 \%$.

\section{Statistische Analysen}

Die statistischen Analysen erfolgten mittels R [41] unter Einbindung der Pakete afex [42] und Ime4 [43]. Fehlende Daten waren durch die Verwendung von Handcomputern zur Durchführung der Testdiagnostik sehr selten ( $0,06 \%)$ und wurden über Bayesianische multiple Imputation [44] geschätzt.

\section{Veränderungsmessung: Statistische und klinische Signifikanz}

Die durch die IES-R erfasste PTBS-Symptombelastung wurde als primäres Outcome-Maß definiert. Waren die Voraussetzungen erfüllt, kamen gemischte lineare Regressionsmodelle mit Freiheitsgradbestimmung nach Kenward-Rogers [42] und statistischer Kontrolle der Kovariablen Geschlecht, Alter und Aufenthaltsdauer zum Einsatz. Erwiesen sich die Messwertdifferenzen der Messwertpaare als nicht normalverteilt, wurde der 2-seitige Wilcoxon-Test angewandt.

Response wurde gemäß des Reliable Change Index (RCI) [45] als unter Berücksichtigung des Messfehlers reliable Symptomreduktion $(p<0,05)$ operationalisiert. Auch wurde überprüft, ob während der Behandlung reliable Verschlechterungen $(p<0,05)$ auftreten. Remission wurde operationalisiert als das gleichzeitige Vorliegen einer Response und eines unauffälligen Entlassscores. Da für den IES-R, den FFA und den DES-T keine Cut-off-Werte vorliegen, konnte Remission hier nicht bestimmt werden. Bei erfüllten Voraussetzungen wurden Prä-Post-Effektstärken (ES) gemäß der Formel von Cohen [46] berechnet.

\section{Prädiktoren des Behandlungserfolges: Konditionaler Entscheidungsbaum}

Der mögliche Zusammenhang von Ausprägungen bestimmter Kovariablen zum Aufnahmezeitpunkt mit der Frage, ob PatientInnen eine Response erzielen, wurde mittels konditionalen Entscheidungsbaumes untersucht. Konditionale Entscheidungsbäume beruhen auf der rekursiven Partitionierung von Daten und dienen der Extraktion von Entscheidungsregeln aus Erfahrungswissen [47]. Sie sind anderen regressionsanalytischen Verfahren in der Identifizierung von Outcome-Prädiktoren insbesondere dann überlegen, wenn von hoher Multikollinearität der potenziellen Prädiktorvariablen ausgegangen werden muss [48]. Der oberste Knoten (Node 1) eines Entscheidungsbaums umfasst die gesamte Stichprobe, die in der Folge in Subgruppen unterteilt wird. Die Auswahl der Kovariablen und der Cut-off-Werte, anhand derer die Stichprobe unterteilt wird, erfolgen dergestalt, dass die Homogenität der abhängigen Variable in den entstandenen Subgruppen maximiert wird. Dieser Prozess wird sukzessive so lange wiederholt, bis ein Modell entstanden ist, das dem Kliniker die wichtigsten signifikanten binären Entscheidungen in der Prognosestellung in Form einer Baumstruktur darstellt. Zwei wesentliche Probleme dieser Methode stellen Overfitting und Verzerrung der Variablenselektion dar, weswegen in der vorliegenden Untersuchung die CTREE-Methode zum Einsatz kam, die ein weitgehend unverzerrtes rekursives Partitionieren erlaubt [49]. Wir definierten Response als abhängige Variable und fügten alle Variablen als Kovariabeln in das CTREE-Modell ein, hinsichtlich derer sich in Voranalysen mittels Welchs t-Tests, exakten $X^{2}$-Tests und Mann-Whitney-U-Tests signifikante Unterschiede zwischen der Response- und der Nonresponse-Gruppe gezeigt hatten. Diese Kovariablen umfassten die Skala Depression der HEALTH-49 $\left(\mathrm{t}_{(147,8)}=-2,03, \mathrm{p}=0,045\right)$, die Skala Somatoforme Beschwerden der HEALTH-49 $\left(t_{(142,9)}=2,37, p=0,019\right)$, die Skala Wohlbefinden der HEALTH-49 $\left(\mathrm{t}_{(148)}=-2,58, \mathrm{p}=0,010\right)$, das Vorliegen einer komplexen dissoziativen Störung $\left(X^{2}{ }_{(1)}=3,91\right.$, $p=0,048)$ sowie den Summenscore des FFA $\left(t_{(139,4)}=1,99\right.$, $p=0,048$ ). Keine signifikanten Unterschiede zwischen den Respondern und Nonrespondern zeigten sich in den Voranalysen hinsichtlich des Geschlechts $\left(x^{2}(1)=0,00, p=0,999\right)$, des Alters $\left(t_{(143)}=-0,46, p=0,646\right)$, des Vorliegens einer Persönlichkeitsstörung $\left(X^{2}(1)=0,16, p=0,692\right)$, des CTQ-Gesamtscores $\left(t_{(125,5)}=\right.$ $-1,03, p=0,307$ ) und aller Subskalen, des IES-R-Gesamtscores $\left(t_{(142,3)}=-0,44, p=0,654\right)$ und aller Subskalen, des HEALTH-49Scores für interaktionelle Schwierigkeiten $\left(\mathrm{t}_{(140,3)}=-0,12\right.$, $p=0,905)$, des HEALTH-49-Scores für Selbstwirksamkeitserleben $\left(t_{(139,1)}=0,83, p=0,408\right)$, des HEALTH-49-Scores für phobische Angst $\left(t_{(140,5)}=1,68, p=0,095\right)$, des HEALTH-49-Scores für soziale Belastung $\left(t_{(140,9)}=0,85, p=0,395\right)$, des HEALTH-49-Scores für soziale Unterstützung $\left(\mathrm{t}_{(139,8)}=0,65, \mathrm{p}=0,515\right)$, des HEALTH-49Scores für Aktivität und Partizipation $\left(t_{(147,3)}=-0,71, p=0,478\right)$ sowie des DES-T Scores $(U=2759, p=0,564)$.

\section{Ergebnisse}

\section{Stichprobe}

Das Durchschnittsalter der 132 Frauen und 18 Männer der Stichprobe lag bei 47,9 Jahren $(S D=10,5) .133$ der PatientInnen (89\%) 
hatten die deutsche Staatsbürgerschaft. Alle PatientInnen schilderten multiple Traumatisierungen in der Kindheit, die mindestens das Kriterium „mittelgradig bis schwer“ des CTQ erfüllten. Die Durchschnittswerte lagen bei 16,8 $(S D=6,8)$ für sexuellen Missbrauch, 14,8 (SD = 6,0) für körperliche Gewalt, 19,1 $(S D=5,3)$ für emotionalen Missbrauch, 14,2 (SD = 4,7) für körperliche Vernachlässigung und 20,7 (SD = 4,8) für emotionale Vernachlässigung.

139 PatientInnen (93\%) wiesen komorbide affektive Störungen auf, 67 PatientInnen (45\%) Angststörungen, 23 PatientInnen (31\%) Zwangsstörungen, 61 PatientInnen (41\%) somatoforme Störungen und 32 PatientInnen (21\%) Essstörungen. Eine komorbide Persönlichkeitsstörung fand sich bei 72 PatientInnen (48\%). Am häufigsten war die Emotional instabile Persönlichkeitsstörung vom Borderline-Typus mit 30 Fällen (20\%), gefolgt von der Ängstlichen Persönlichkeitsstörung mit 15 Fällen (10\%). 31 Patientlnnen (21\%) wiesen eine DDNOS auf, 8 PatientInnen (5\%) eine DIS. 108 PatientInnen (72\%) nahmen zum Aufnahmezeitpunkt Antidepressiva ein, 51 PatientInnen (34\%) Anxiolytika, 69 PatientInnen (46\%) Antipsychotika und 93 PatientInnen (62\%) Analgetika. Bei 63 PatientInnen (42\%) fand sich mindestens ein fremdanamnestisch bestätigter Suizidversuch in der Anamnse. Alle PatientInnen befanden sich aktuell in ambulanter Psychotherapie, 58\% seit insgesamt mehr als 160 Stunden. Stationär-psychiatrische und -psychosomatische Behandlungen fanden sich bei 132 PatientInnen (88\%). Der Median in Bezug auf die Anzahl stationärer Vorbehandlungen betrug 3 $(\min =0 ; \max =42)$.

\section{Veränderungsmessung: statistische und klinische Signifikanz}

Die signifikante Verbesserung der PTBS-Symptomatik vom Aufnahmezeitpunkt t1 zum Entlasszeitpunkt $t 2\left(\mathrm{~F}_{(1,149)}=112,2, \mathrm{p}<0,0001\right)$ entsprach einem großen Effekt $(d=1,8)$ und bedeutete für $52 \%$ der PatientInnen eine Response. Gleichzeitig beschrieben 3,8\% der PatientInnen eine reliable Verschlechterung. Auch bezüglich weiterer Symptombereiche wie Depressivität $\left(F_{(1,149)}=133,1, p<0,0001\right.$, $69 \%$ Response, $9 \%$ Remission) und somatoformer Beschwerden $\left(F_{(1,149)}=39,1, p<0,0001,41 \%\right.$ Response, $7 \%$ Remission $)$ ließen sich mittlere bis hohe Effekte erzielen ( $>$ Tab. 1). Darüber hinaus schilderten die PatientInnen zum Entlasszeitpunkt ein signifikant besseres Wohlbefinden $\left(F_{(1,149)}=114,8, p<0,0001,50 \%\right.$ Response, $14 \%$ Remission), mehr Achtsamkeit $\left(F_{(1,149)}=51,5, p<0,0001,20 \%\right.$ Response), geringere interaktionelle Schwierigkeiten $\left(F_{(1,149)}=66,0\right.$, $\mathrm{p}<0,0001,54 \%$ Response, $8 \%$ Remission) sowie ein verbessertes Selbstwirksamkeitserleben $\left(F_{(1,149)}=59,8, p<0,0001,47 \%\right.$ Response, $13 \%$ Remission).

\section{Prädiktoren des Behandlungsverlaufs: Konditionaler Entscheidungsbaum}

Mittels CTREE-Modell konnte ein Entscheidungsbaum mit 3 Prädiktoren identifiziert werden ( $\triangleright$ Abb. 1). Die Basisrate einer Response bezüglich der PTBS-Belastung lag in der Gesamtstichprobe (Node 1; $N=150$ ) bei $52 \%$. Die erste Unterteilung in Subgruppen erfolgte anhand der bei Aufnahme im HEALTH-49 geschilderten somatoformen Beschwerden ( $p<0,01)$. In der Subgruppe der PatientInnen mit Scores für somatoforme Beschwerden über 3,29 (Node 7; $=29$ ) sank die Rate reliabler Verbesserungen auf 27,6\% ab und halbierte sich somit fast. In der Subgruppe von PatientIn- nen mit Scores für somatoforme Beschwerden unter 3,29, bei denen eine komplexe dissoziative Störung diagnostiziert wurde (Node 6; $\mathrm{N}=33$ ), lag die Rate von PatientInnen mit Response mit einem Wert von 39,4\% ebenfalls unter dem Durchschnitt $(p<0,05)$. PatientInnen, die keine komplexe dissoziative Störung und Scores für somatoforme Beschwerden unter 3,29 aufwiesen (Node 3; $\mathrm{N}=88$ ) konnten abermals signifikant in Subgruppen unterschieden werden $(p<0,01)$. PatientInnen mit Scores von 52 oder darunter im FFA (Node 4; $\mathrm{N}=18$ ) wiesen eine Responsewahrscheinlichkeit von 38,9\% auf. Jene PatientInnen hingegen, deren Score im FFA über dem Wert von $52 \mathrm{lag}$ (Node 5; $N=70$ ), wiesen eine Responsewahrscheinlichkeit von $71,4 \%$ auf.

\section{Diskussion}

\section{Einordnung der Ergebnisse}

Mit der vorliegenden retrospektiven Untersuchung naturalistischer Therapieverläufe sollten Symptomverläufe von PatientInnen mit komplexer PTBS nach schweren (in der Regel sexualisierten) Gewalterfahrungen in der Kindheit analysiert und Prädiktoren einer Response identifiziert werden. Eine geringe Abbruchrate und seltene reliable Verschlechterungen spiegeln eine hohe Sicherheit und gute Akzeptanz der Behandlung wider. Es fanden sich große Effekte hinsichtlich der PTBS-Kernsymptomatik, Depressivität und Wohlbefinden sowie mittlere Effekte hinsichtlich Angst, somatoformer Beschwerden, interaktioneller Schwierigkeiten, Selbstwirksamkeit und Achtsamkeit. Die Ergebnisse sind damit vergleichbar mit jenen aus randomisiert-kontrollierten Studien zu traumafokussierter Therapie mit ähnlichen Stichproben $[16,17,19]$ und besser als die Ergebnisse, die für ein primär stabilisierendes Vorgehen berichtet werden. So fanden Lampe et al. [11] für die Psychodynamisch Imaginative Traumatherapie (PITT) keine bis geringe Effekte hinsichtlich der PTBS-Kernsymptomatik [13]. Die Abbruchrate lag dabei trotz des „stabilisierenden“ Vorgehens mit 13\% höher als in der vorliegenden Untersuchung. Jepsen et al. [51] berichten für ihr auf Psychoedukation und Gruppentherapie beruhendes Behandlungskonzept für PatientInnen mit PTBS infolge von sexuellem Missbrauch in der Kindheit geringe bis mittlere Effekte hinsichtlich Dissoziation ( $d=0,3)$, Depressivität $(d=0,4)$, PTBS $(d=0,6)$ und interpersonellen Schwierigkeiten $(d=0,4)$. Die Abbruchrate lag in dieser Untersuchung bei 5,4\% und damit auf einem ähnlichen Niveau wie in der vorliegenden Untersuchung (7\%). Damit legen unsere Ergebnisse nahe, dass traumafokussierte Therapie mit PatientInnen mit komplexer PTBS nach schwerer (sexualisierter) Gewalt in der Kindheit auch unter klinischen Routinebedingungen sicher und wirksam durchführbar ist.

\section{Zur Bedeutung der Prädiktoren}

Die Ergebnisse untermauern Befunde, dass Dissoziation einen negativen Einfluss auf den Therapieverlauf von PatientInnen mit PTBS hat und Anpassungen des therapeutischen Vorgehens nötig macht $[50,51]$. Gleichzeitig stehen die vorliegenden Ergebnisse auch mit Befunden im Einklang, die darauf hinweisen, dass ein traumafokussiertes Vorgehen in der Therapie geeignet ist, dissoziative Symptome zu reduzieren [52]. Mit somatoformen Beschwerden konnten wir darüber hinaus einen Prädiktor für die Behandlung der PTBS 


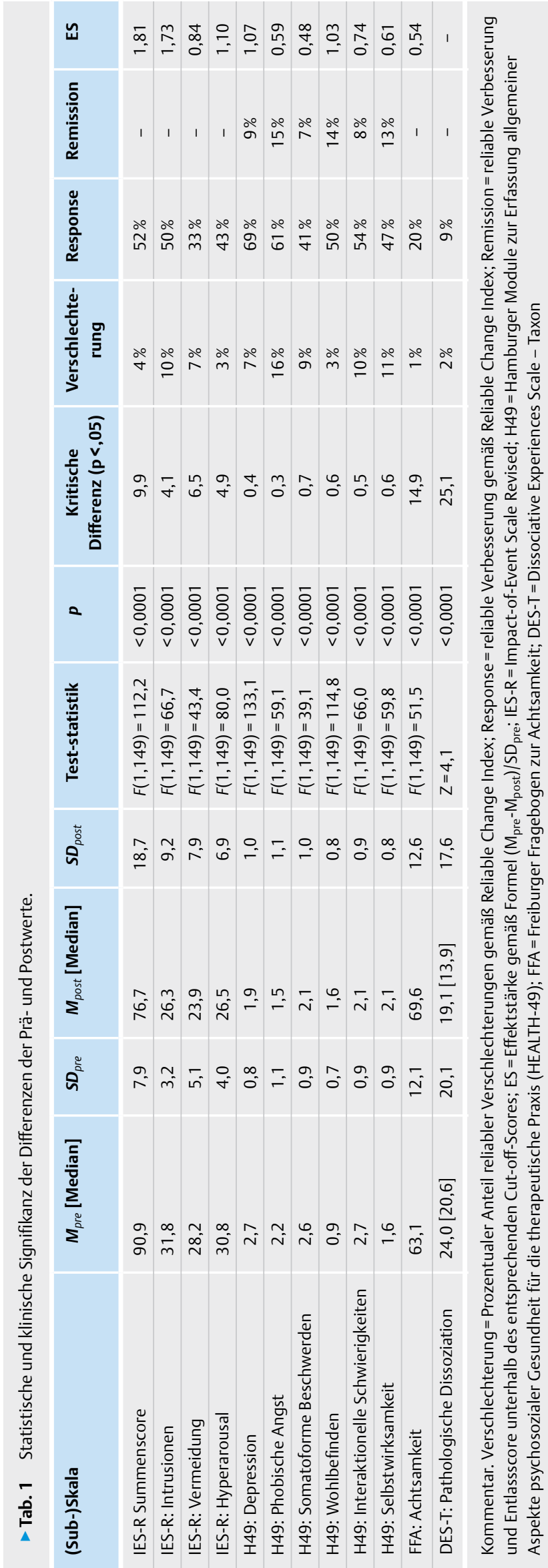

identifizieren, dessen Bedeutung im Zusammenhang mit Traumafolgestörungen bis heute vernachlässigt wird [53]. Schließlich konnte mit der vorliegenden Untersuchung - unseres Wissens erstmalig - gezeigt werden, dass Achtsamkeitsdefizite die Behandlung der komplexen PTBS merklich beeinträchtigen können. Dies erscheint umso bemerkenswerter, als dass Achtsamkeit zwar einen Baustein zahlreicher etablierter Manuale zur Behandlung der PTBS darstellt (z. B. $[12,16])$ und auch Teil des untersuchten Behandlungsprogrammes ist, die Rolle von Achtsamkeitsdefiziten für die Entstehung und Aufrechterhaltung posttraumatischer Belastungsstörungen jedoch weitgehend ungeklärt ist.

Es liegen Befunde vor, dass Achtsamkeit den Zusammenhang von traumatischen Erfahrungen in der Kindheit und späterer PTBSSymptomatik mediiert [54]. Achtsamkeit scheint die negative Wirkung von dysfunktionalen Kognitionen abzuschwächen, während Achtsamkeitsdefizite mit Störungen der Emotionsregulation sowie Vermeidungsverhalten assoziiert sind [55, 56]. Zugleich weisen Achtsamkeitsdefizite auch einen Zusammenhang mit Neurotizismus auf [57]. Weitere Untersuchungen müssen daher klären, ob es sich bei Achtsamkeitsdefiziten um ein Epiphänomen handelt. Die Frage, welchen Anteil Achtsamkeitstrainings am Behandlungserfolg haben, sollte mittels Dismantling-Studien geprüft werden.

\section{Zum Umgang mit Nonresponse}

Bei PatientInnen mit komplexer PTBS gelten Nonresponseraten von $50 \%$ als nicht außergewöhnlich und es werden Drop-out-Raten von $50 \%$ und mehr beobachtet $[10,58]$. Auch sind Überlebende sexualisierter Gewalt besonders gefährdet, während stationärer Therapie Verschlechterungen ihres Zustandes zu erleiden [59]. Vor diesem Hintergrund ist die berichtete Responserate von 52 \% in der hier untersuchten, besonders schwer belasteten Gruppe von PatientInnen, einerseits als positiv zu bewerten. Die Abbruchrate im Untersuchungszeitraum von nur 7 \% darf zudem als außergewöhnlich niedrig bezeichnet werden. Auch liegen die im Rahmen der vorliegenden Untersuchung festgestellten Raten reliabler Verschlechterungen im Vergleich zur in der Literatur berichteten Werten [60] im unterdurchschnittlichen Bereich. Andererseits zeigt sich deutlich die Schwierigkeit, auch mit leitliniengerechter Therapie allen PatientInnen eine Response zu ermöglichen. Insbesondere in Verbindung mit komorbiden dissoziativen Störungen sind die Störungsbilder von PatientInnen mit komplexer PTBS oftmals als chronische und schwere psychische Erkrankung zu verstehen [61] und selbst hochwirksame Behandlungsprogramme führen angesichts der Schwere der Störungen bei einer erheblichen Anzahl von PatientInnen zu keiner Remission. Langfristig angelegte Behandlungspläne müssen demnach auch Überlegungen beinhalten, PatientInnen in einem Leben mit chronisch eingeschränktem Funktionsniveau zu unterstützen und Kontakte zu Unterstützungsangeboten zu vermitteln, die die soziale Teilhabe verbessern können.

Andererseits spricht zunehmende empirische Evidenz dafür, dass die Raten von Nonresponse und reliabler Verschlechterungen, die auch bei sorgfältiger Dissemination leitliniengerechter Behandlungsprogramme beobachtet werden, womöglich über systematisches Therapie-Monitoring und Feedback reduzieren lassen. Therapiemonitoring ermöglicht es, problematische Verläufe frühzeitig zu erkennen, gegenzusteuern und so Abbrüche zu verhindern und Responseraten zu verbessern [62]. 


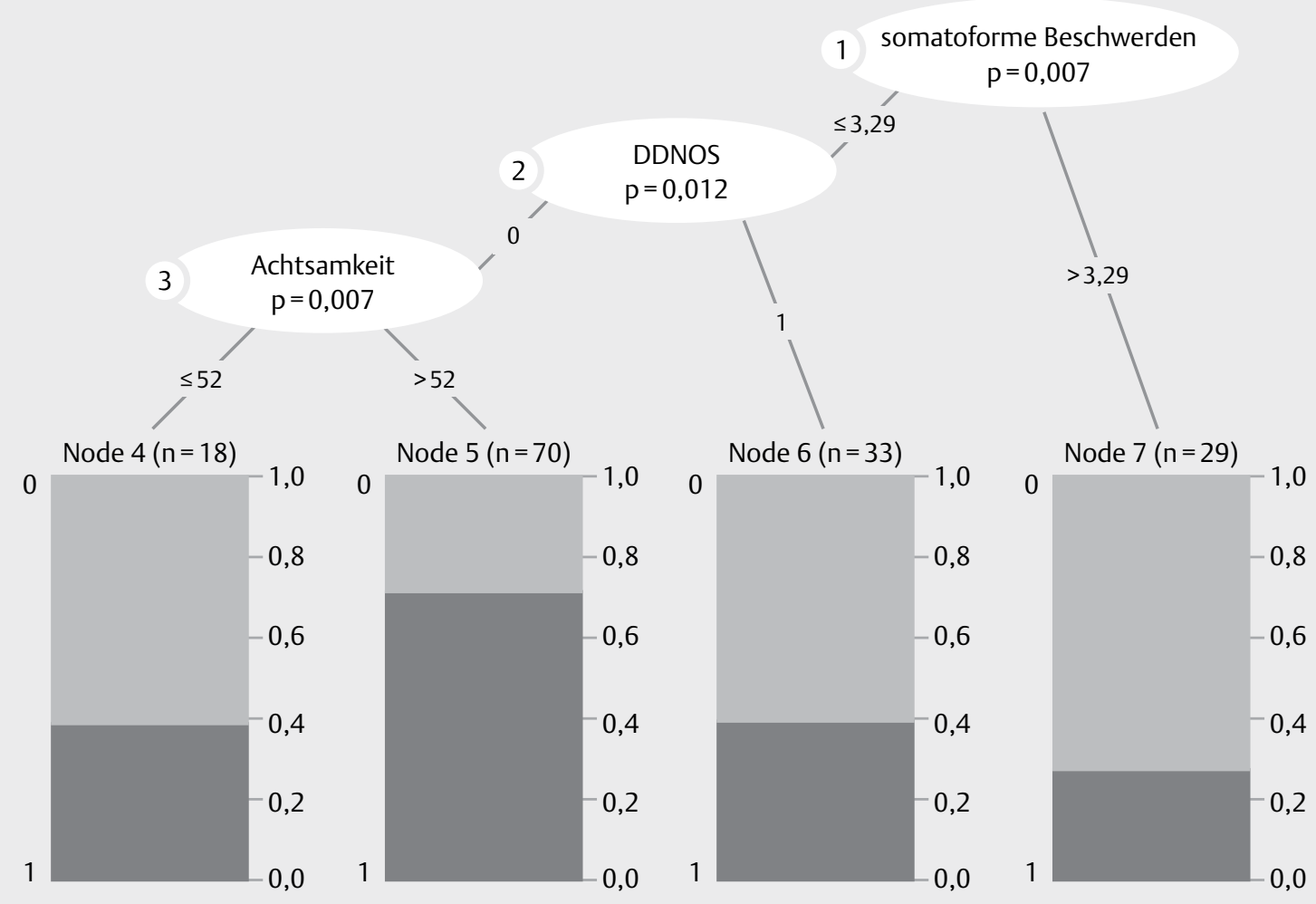

- Abb. 1 Das Entscheidungsbaummodell zeigt, anhand welcher Prädiktoren und Cut-Off-Werte bereits bei Aufnahme signifikant die Wahrscheinlichkeit einer reliablen Verbesserung („1“; dunkelgrau) sowie eines Persistierens der PTBS-Belastung („0“; hellgrau) bestimmt werden kann. Die bedingten Wahrscheinlichkeiten P(Response | Bedingung) sind den rechten y-Achsen der Diagramme zu entnehmen. Somatoforme Beschwerden, das Vorliegen einer komplexen dissoziativen Störung (DDNOS oder DIS) und Achtsamkeit erweisen sich als signifikante Prädiktoren des Behandlungsverlaufs.

\section{Stärken und Schwächen der Untersuchung}

Aus dem naturalistischen Charakter der Untersuchung ergeben sich spezifische Stärken und Schwächen. So stehen der hohen ökologischen Validität bspw. das Problem einer fehlenden Einschätzung der Schwere der PTBS sowie komorbider Störungen durch strukturierte Interviewverfahren gegenüber. Zukünftige Untersuchungen sollten darüber hinaus nicht nur die Aufenthaltsdauer, sondern auch den exakten Zeitumfang der Traumakonfrontation erfassen und den möglichen Einfluss dieser Kovariablen auf den Behandlungsverlauf untersuchen. Die Vermutung liegt nahe, dass der Umfang der Traumakonfrontation eine bedeutende Einflussgröße darstellt, was in der vorliegenden Untersuchung jedoch keine ausreichende Berücksichtigung fand. Die größte Einschränkung ergibt sich jedoch aus dem Fehlen einer Katamneseuntersuchung. Somit bleibt unklar, inwiefern die PatientInnen langfristig von der Behandlung profitierten und ob vereinzelte reliable subjektive Verschlechterungen etwa der Angstsymptomatik wie vermutet nur passagere durch die Reduktion von Vermeidung induzierte Phänomene darstellen.

\section{FAZIT FÜR DIE PRAXIS}

Traumafokussierte Psychotherapie ist auch unter naturalistischen Bedingungen mit in der Kindheit schwer sexuell oder körperlich traumatisierten PatientInnen mit komplexer PTBS sicher durchführbar. Geringe Abbruch- und Verschlechterungsraten spiegeln Sicherheit und Akzeptanz eines traumafokussierten Vorgehens wider. Gleichzeitig ist zu beobachten, dass einzelne Subgruppen wie PatientInnen mit ausgeprägten somatoformen Beschwerden, komplexen dissoziativen Störungen oder Achtsamkeitsdefiziten schlechter auf die Behandlung ansprechen.

\section{Danksagung}

Unser Dank gilt den PatientInnen, Co-TherapeutInnen und TherapeutInnen der Klinik St. Irmingard. 
Interessenkonflikt

Leonhard Kratzer, Peter Heinz, Rebecca Schennach, Frank Padberg und Andrea Jobst geben an, dass kein Interessenkonflikt besteht. Günter Schiepek gibt an, dass er finanzielle Verbindungen zur Center for Complex Systems GmbH \& Co. KG, die das Therapieprozessmonitoring-Tool Synergetic Navigation System (SNS) vertreibt, hat.

\section{Literatur}

[1] World Health Organization. International Statistical Classification of Diseases and Related Health Problems. 10 $0^{\text {th }}$ revision Geneva: World Health Organisation; 1992

[2] Herman JL. Complex PTSD: A syndrome in survivors of prolonged and repeated trauma. J Trauma Stress 1992; 5: 377-391

[3] Pelcovitz D, van der Kolk B, Roth S et al. Development of a criteria set and a structured interview for disorders of extreme stress (SIDES). J Trauma Stress 1997; 10: 3-16

[4] Dorrepaal E, Thomaes K, Hoogendoorn AW et al. Evidence-based treatment for adult women with child abuse-related Complex PTSD: A quantitative review. Eur J Psychotraumatol 2014; 5: 23613 http:// dx.doi.org/10.3402/ejpt.v5.23613

[5] Knefel M, Tran US, Lueger-Schuster B. The association of posttraumatic stress disorder, complex posttraumatic stress disorder, and borderline personality disorder from a network analytical perspective. J Anxiety Disord 2016; 43: 70-78

[6] Maercker A, Brewin CR, Bryant RA et al. Proposals for mental disorders specifically associated with stress in the International Classification of Diseases-11. Lancet 2013; 381: 1683-1685

[7] Spinazzola J, Blaustein M, van der Kolk BA. Posttraumatic Stress Disorder Treatment Outcome Research: The Study of Unrepresentative Samples? J Trauma Stress 2005; 18: 425-436

[8] Corrigan FM, Hull AM. Neglect of the complex: why psychotherapy for post-traumatic clinical presentations is often ineffective. Psychiatr Bull 2015; 39: 86-89

[9] Ford JD, Kidd TP. Early childhood trauma and disorders of extreme stress as predictors of treatment outcome with chronic posttraumatic stress disorder. J Trauma Stress 1998; 11: 743-761

[10] Schottenbauer MA, Glass CR, Arnkoff DB et al. Nonresponse and dropout rates in outcome studies on PTSD: review and methodological considerations. Psychiatry 2008; 71: 134-168

[11] Lampe A, Mitmansgruber $\mathrm{H}$, Gast $\mathrm{U}$ et al. Therapieevaluation der Psychodynamisch Imaginativen Traumatherapie (PITT) im stationären Setting. Neuropsychiatr 2008; 22: 189-197

[12] Reddemann L. Psychodynamisch Imaginative Traumatherapie. PITT Das Manual. Stuttgart: Klett-Cotta; 2011

[13] Priebe K, Steil R, Kleindienst $N$ et al. Psychotherapie der Posttraumatischen Belastungsstörung nach sexuellem Missbrauch: Ein Überblick über die Datenlage. Psychother Psychosom Med Psychol 2012; 62: 5-17

[14] Rosner R, Henkel C, Ginkel K et al. Was passiert nach der stationären Stabilisierung mit komplex traumatisierten PTB-Patientinnen? Die Bedeutung von Stabilisierung und Konfrontation für die Behandlung traumatisierter Frauen. Z Klin Psychol Psychiatr Psychother 2010; 58 : 127-135

[15] De Jongh A, Resick PA, Zoellner LA et al. Critical analysis of the current treatment guidelines for complex PTSD in adults. Depress Anxiety 2016; 33: 359-369

[16] Bohus M, Dyer A, Priebe K et al. Dialectical Behaviour Therapy for Post-traumatic Stress Disorder after Childhood Sexual Abuse in Patients with and without Borderline Personality Disorder: A Randomised Controlled Trial. Psychother Psychosom 2013; 82: 221-233
[17] Harned MS, Korslund KE, Linehan MM. A pilot randomized controlled trial of Dialectical Behavior Therapy with and without the Dialectical Behavior Therapy Prolonged Exposure protocol for suicidal and self-injuring women with borderline personality disorder and PTSD. Behav Res Ther 2014; 55: 7-17

[18] van den Berg DG, de Bont PM, van der Vleugel BM et al. Prolonged exposure vs. eye movement desensitization and reprocessing vs. waiting list for posttraumatic stress disorder in patients with a psychotic disorder: A randomized clinical trial. JAMA Psychiatry 2015; 72: 259-267

[19] Ehring T, Welboeren R, Morina $\mathrm{N}$ et al. Meta-analysis of psychological treatments for posttraumatic stress disorder in adult survivors of childhood abuse. Clin Psychol Rev 2014; 34: 645-657

[20] Dell PF. A New Model of Dissociative Identity Disorder. Psychiatr Clin North Am 2006; 29: 1-26

[21] van der Hart O, Groenendijk M, Gonzalez A et al. Dissociation of the Personality and EMDR Therapy in Complex Trauma-Related Disorders: Applications in Phases 2 and 3 Treatment. J EMDR Pract Res 2014; 8: $33-48$

[22] Cloitre M, Stovall-McClough KC, Nooner K et al. Treatment for PTSD related to childhood abuse: A randomized controlled trial. Am J Psychiatry 2010; 167: 915-924

[23] Heinz P, Pfitzer F. Störungsspezifisches stationäres Behandlungsprogramm für komplex traumatisierte Erwachsene: Konzept der Traumastation der Klinik St. Irmingard in Prien am Chiemsee. Trauma - Zeitschrift für Psychotraumatologie und ihre Anwendungen 2014; 12: $26-37$

[24] Bohus M, Wolf-Arehult M. Interaktives Skillstraining für BorderlinePatienten. (2. Auflage) Stuttgart: Schattauer; 2013

[25] Priebe K, Schmahl C, Stiglmayr C. Dissoziation - Theorie und Therapie. Berlin: Springer; 2013

[26] Shapiro F. Eye Movement Desensitization and Reprocessing: Basic principles, protocols, and procedures. $2^{\text {nd }}$ ed. New York: Guilford Press; 2001

[27] Haken H, Schiepek G. Synergetik in der Psychologie. Selbstorganisation verstehen und gestalten. Göttingen: Hogrefe; 2010

[28] Schiepek G, Aichhorn W, Gruber M et al. Real-Time Monitoring of Psychotherapeutic Processes: Concept and Compliance. Front Psychol 2016; 7: 604 https://doi.org/10.3389/fpsyg.2016.00604

[29] Bernstein DP, Fink L. Childhood Trauma Questionnaire. A retrospective self-report. San Antonio: The Psychological Corporation; 1998

[30] Wingenfeld K, Spitzer C, Mensebach C et al. Die deutsche Version des Childhood Trauma Questionnaire (CTQ): Erste Befunde zu den psychometrischen Kennwerten. Psychother Psychosom Med Psychol 2010; 60: 442-450

[31] Weiss DS, Marmar CR. The Impact of Event Scale - Revised. in Wilson JP, Keane TM. (eds). Assessing psychological trauma and PTSD. New York: Guilford Press; 1996: 399-411

[32] Maercker A, Schützwohl M. Erfassung von psychischen Belastungssfolgen: Die Impact of Event Skala-revidierte Version. Diagnostica 1998; 44: $130-141$

[33] Rosner R, Hagl M. Die revidierte Impact of Event-Skala (IES-R). Psychosomatik und Konsiliarpsychiatrie 2008; 2: 240-243

[34] Walach H, Buchheld N, Buttenmüller $V$ et al. Measuring mindfulness The Freiburg Mindfulness Inventory (FMI). Pers Individ Dif 2006; 40: 1543-1555

[35] Waller N, Putnam FW, Carlson EB. Types of dissociation and dissociative types: A taxometric analysis of dissociative experiences. Psychol Methods 1996; 1: 300-321

[36] Spitzer C, Freyberger H, Brähler E et al. Teststatistische Überprüfung der Dissociative Experiences Scale-Taxon (DES-T). Psychother Psych Med 2015; 65: 134-139 
[37] Rabung S, Harfst T, Kawski S et al. Psychometrische Überprüfung einer verkürzten Version der Hamburger Module zur Erfassung allgemeiner Aspekte psychosozialer Gesundheit für die therapeutische Praxis (HEALTH-49). Z Psychosom Med Psychother 2009; 55: 162-179

[38] Boroske-Leiner K, Hofmann A, Sack M. Ergebnisse zur internen und externen Validität des Interviews zur komplexen posttraumatischen Belastungsstörung (I-kPTBS). Psychother Psychosom Med Psychol 2008; 58: 192-199

[39] Overkamp B. Differentialdiagnostik der dissoziativen Identitätsstörung (DIS) in Deutschland [Dissertation]. München: Ludwig-MaximiliansUniversität; 2005

[40] Ross CA. The dissociative disorders interview schedule: A structured interview. Dissociation 1989; 2: 169-189

[41] R Core Team. R: A Language and Environment for Statistical Computing. 2017; https://www.R-project.org

[42] Singmann H, Bolker B, Westfall J et al. afex: Analysis of Factorial Experiments. R package version 0.17-8. 2017. https://CRAN.R-project. org/package $=$ afex

[43] Bates D, Mächler M, Bolker B et al. Fitting Linear Mixed-Effects Models Using Ime4. J Stat Softw doi:10.18637/jss.v067.i01

[44] Su YS, Gelman A, Hill j et al. Multiple Imputation with Diagnostics (mi) in R: Opening Windows into the Black Box. J Stat Softw 2011; 45: 1-31

[45] Jacobson NS, Follette WC, Revenstorf D. Psychotherapy outcome research: methods for reporting variability and evaluating clinical significance. Behav Ther 1984; 15: 336-352

[46] Cohen J. Statistical power analysis for behavioural sciences. Hillsdale: Erlbaum; 1988

[47] Breiman L, Friedman JH, Olshen RA et al. Classification and regression trees. New York: Chapman \& Hall; 1984

[48] Speybroeck N. Classification and regression trees. Int J Public Health 2011; 57: 243-246

[49] Hothorn T, Zeileis A. partykit: A Modular Toolkit for Recursive Partitioning in R. J Mach Learn Res 2015; 16: 3905-3909

[50] Bae H, Kim D, Park YC. Dissociation Predicts Treatment Response in Eye Movement Desensitization and Reprocessing (EMDR) for Post-traumatic Stress Disorder. J Trauma Dissociation 2016; 17: $112-130$

[51] Jepsen EKK, Langeland W, Sexton $\mathrm{H}$. Inpatient treatment for early sexually abused adults: A naturalistic 12-month follow-up study. Psychol Trauma 2014; 6: 142-151
[52] Harned MS, Gallop R], Valenstein-Mah HR. What changes when? The course of improvement during a stage-based treatment for suicidal and self-injuring women with borderline personality disorder and PTSD. Psychother Res http://dx.doi.org/10.1080/10503307.2016.125 2865

[53] McFarlane AC. Post-traumatic stress disorder is a systemic illness, not a mental disorder: is Cartesian dualism dead? Med J Aust 2017; 206: 248-249

[54] Kratzer L, Heinz P, Pfitzer F et al. Mindfulness and pathological dissociation fully mediate the association of childhood abuse and PTSD symptomatology. Eur J Trauma Dissociation 2018; 2: 5-10

[55] Glück TM, Tran US, Raninger $S$ et al. The influence of sense of coherence and mindfulness on PTSD symptoms and posttraumatic cognitions in a sample of elderly Austrian survivors of World War II. Int Psychogeriatr 2016; 28: 435-441

[56] Ehring T, Ehlers A. Does rumination mediate the relationship between emotion regulation ability and posttraumatic stress disorder? Eur J Psychotraumatol doi:10.3402/ejpt.v5.23547

[57] Giluk TL. Mindfulness, Big Five personality, and affect: A meta-analysis. Pers Infivid Dif 2009; 47: 805-811

[58] McDonagh A, Friedman M, McHugo G et al. Randomized trial of cognitive-behavioral therapy for chronic posttraumatic stress disorder in adult female survivors of childhood sexual abuse. J Consult Clin Psychol 2005; 73: 515-524

[59] Spitzer C, Barnow S, Freyberger H] et al. Symptomverschlechterungen während stationärer Psychotherapie - Wer ist betroffen? Die Psychodynamische Psychotherapie 2008; 7: 3-15

[60] Lambert M]. The Efficacy and Effectiveness of Psychotherapy. in Lambert MJ. (ed). Bergin and Garfield's Handbook of Psychotherapy and Behavior Change. Volume 6: New York: John Wiley \& Sons; 2013: 169-218

[61] Vazquez AIG, Ameneiros NS, del Valle JCD et al. Revisiting the concept of severe mental illness: severity indicators and healthcare spending in psychotic, depressive and dissociative disorders. J Ment Health doi:10.1080/09638237.2017.1340615

[62] Lambert M]. Maximizing Psychotherapy Outcome beyond EvidenceBased Medicine. Psychother Psychosom 2017; 86: 80-89 